\section{Carotid intima media thickness in patients with lupus nephritis: some appraisals}

\author{
V. Bruzzese, C. Hassan, A. Zullo \\ Internal Medicine, Rheumatology and Gastroenterology, 'Nuovo Regina Margherita' Hospital, Rome, Italy
}

W e read with great interest the study by Shaharir et al. which found that 6 $(15.4 \%)$ out of 39 patients with controlled lupus nephritis (LN) had increased carotid intima media thickness (CIMT), and that CIMT values were independently associated with older age at diagnosis and proteinuria at multivariate analysis (1). We would comment on some aspects of this study.

First: The estimation of increased CIMT rate $(15.4 \%)$ in $\mathrm{LN}$ patients would appear questionable, since an appropriate control group is lacking in the study. Indeed, for their Asian patients (mean age: $35.2 \pm 11.2$ years), the Authors used the as normal (0.64-0.66 mm) the CIMT values of $<50$ years Caucasian subjects studied in Germany (2). Therefore, the estimation would appear misleading.

Second: Some relevant concerns arise in the interpretation of logistic regression analysis results, which were based on only 6 patients with increased CIMT values. For instance, it is unreliable that the regression accurately detected a different prevalence of cardiovascular risk $(\mathrm{CV})$ factors between such a very small patient group and the remaining cases. In addition, it would appear controversial the finding that patient age at diagnosis - but not the current age - was an independent predictor of increased CIMT. In fact, despite we failed to understand the correct mean age of patients, the current age of the 6 patients with increased CIMT was significantly higher than that of other patients with normal CMIT values, as reported either in Table
I $(47.5 \pm 7.7$ vs $33.0 \pm 10.3 ; \mathrm{P}<0.01)$ or in the text $(42.4 \pm 7.8$ years vs $31.9 \pm 9.5$ years; $\mathrm{P}<0.01)$. Since the disease duration was similar in both patient groups $(7.9 \pm 5.9 v s$ $8.4 \pm 4.6 ; \mathrm{P}=0.86$ ), the same gap of age was present at $\mathrm{LN}$ diagnosis.

Consequently, it remains unclear how the regression analysis would have found significant the difference of age at LN diagnosis, but not the same difference of age currently present between patient groups. The increase of CIMT values with patient age widely recognized in literature further questions on the reliability of a logistic regression analysis based on only 6 cases $(2,3)$.

Third. Surprisingly, the actual values in the 6 patients with increased CIMT is lacking, even if it was stated that, overall, the median of CMIT values was $0.8 \mathrm{~mm}$ in male and 0.5 in female patients. Generally, CIMT values $<0.9 \mathrm{~mm}$ were considered normal, values between 0.9 and $1.2 \mathrm{~mm}$ as moderate thickness, and values $>1.2 \mathrm{~mm}$ as pre-clinical atherosclerosis (3). Therefore, it would have been more clinically relevant to assess whether pre-clinical atherosclerosis (CMIT>1.2 $\mathrm{mm}$ ) prevalence is really increased in $\mathrm{LN}$ patients as compared to matched controls, to prompt target therapies in these patients. With this aim, the CIMT values of LN patients without any traditional $\mathrm{CV}$ risk factor should be compared with that of age-, sex-, and racematched subjects without $\mathrm{CV}$ risk factors to disclose whether $\mathrm{LN}$ is an independent risk factor for $\mathrm{CV}$ events (3). $\overline{\text { Corresponding author: }}$ Vincenzo Bruzzese Medicina Interna Reumatologia e Gastroenterologia Ospedale Nuovo Regina Margherita Via E. Morosini, 30 - 00153 Roma, Italia E-mail: vinbruzzese@tin.it 


\section{REFERENCES}

1. Shaharir SS, Said MMS, Kong NC. Predictors of thickened carotid intima media thickness among well controlled lupus nephritis patients in a Malaysian tertiary center. Reumatismo. 2012; 64: 341-9.

2. Lorenz MW, von Kegler S, Steinmetz H, et al. Carotid intima-media thickening indicates a higher vascular risk across a wide age range: prospective data from the Carotid Atherosclerosis Progression Study (CAPS). Stroke. 2006; 37 : 87-92.

3. Bruzzese V, Marrese C, Zullo A, et al. Carotid artery intima-media thickness in patients with autoimmune connective tissue diseases: a casecontrol study. Intern Emerg Med. 2011 Oct 28. [Epub ahead of print] 